\title{
'Capsula formativa' para el reconocimiento de alteraciones y patrón de marcha en un paciente con la enfermedad de Parkinson
}

\author{
Lidia Castillo-Mariqueo y Lydia Giménez-Llort \\ Universidad Autónoma de Barcelona (España)
}

\begin{abstract}
Los trastornos de la marcha tienen un gran impacto en la población adulto mayor a nivel mundial. En este grupo etario la alteración de la marcha se puede complicar con el síndrome geriátrico de caída. Los objetivos son reconocimiento de las principales alteraciones en la locomoción y el desplazamiento de un adulto mayor. El método se basa en una sesión teórico-práctica de tres horas de duración donde participaron 36 personas fue aplicada una encuesta inicial y final para detectar los principales indicadores de reconocimiento visual de posibles alteraciones. Se utilizaron registros audiovisuales de casos clínicos bajo diferentes planos de observación. Posteriormente se llevó a cabo una discusión para la unificación de las principales ideas junto a un diagnóstico final. Los resultados son: en el ámbito conceptual los sujetos no alcanzan el 50\% de reconocimiento de términos clave. Respecto a la identificación de alteraciones y cambios producidos por el envejecimiento sólo el $32 \%$ pudo determinar los elementos de mayor relevancia, así como factores intrínsecos y extrínsecos que estuvo por debajo del $30 \%$. Al finalizar, los participantes fueron capaces de reconocer sobre el $70 \%$ de elementos claves, alcanzando una diferencia estadística significativa de $p=0,000\left(\mathrm{X}^{2}\right)$. Se mejoró significativamente la capacidad de reconocimiento de alteraciones de la locomoción y el desplazamiento de las personas mayores.
\end{abstract}

Palabras clave: Envejecimiento, Locomoción, Desplazamiento, Gerontología, Geriatría.

'Formative capsule' for the recognition of alterations and gait pattern in a patient with Parkinson's disease. Gait disorders have a great impact on the elderly population worldwide. In this age group, gait disturbance can be complicated by geriatric fall syndrome. The objective is Recognition of the main changes in locomotion and displacement of an older adult. The method is based on a theoretical and practical session of three hours in which 36 people participated was applied an initial and final survey to detect the main indicators of visual recognition of possible alterations. Audiovisual records of clinical cases were used under different observation planes. Subsequently, a discussion was held to unify the main ideas together with a final diagnosis. The results are in the conceptual field, the subjects do not reach 50\% recognition of key terms. Regarding the identification of alterations and changes produced by aging, only $32 \%$ were able to determine the most relevant elements, as well as intrinsic and extrinsic factors that were below $30 \%$. Upon completion, participants were able to recognize over $70 \%$ of key elements, reaching a significant statistical difference of $p=0.000\left(\mathrm{X}^{2}\right)$. The ability to recognize alterations in locomotion and displacement of the elderly was significantly improved.

Keywords: Aging, Locomotion, Displacement, Gerontology, Geriatrics.

Correspondencia: Lidia Castillo-Mariqueo. Universidad Autónoma de Barcelona, Facultad de Medicina. Av. de Can Domènech, s/n. C.P.: 08193. Bellaterra, Barcelona (España). E-mail: lidia.castillom@e-campus.uab.cat 
La enfermedad de Parkinson (EP) constituye la segunda enfermedad neurodegenerativa más frecuente después de la demencia tipo Alzheimer. Corresponde a un trastorno neurodegenerativo, progresivo y multisistémico que aumenta su incidencia y prevalencia durante el envejecimiento. Posee una distribución universal, afectando por igual tanto a hombres como a mujeres (Suzuki et al., 2017; Sveinbjornsdottir, 2016).

En su cuadro clínico de la EP se caracteriza por signos y síntomas de tipo motor, y no motor. Entre los signos y síntomas motores se encuentran: temblor en reposo, rigidez, lentitud para iniciar los movimientos (Bradicinesia), inestabilidad postural, y alteraciones de la locomoción y el desplazamiento. Respecto a los signos y síntomas no motores, existe una gran variedad de ellos, destacando los trastornos neuropsiquiátricos que pueden ser: depresión, ansiedad, apatía, alucinaciones y delirios, así como disfunción autonómica, trastornos del sueño y síntomas gastrointestinales (Jankovic, 2008; Martínez-Fernández, Gasca-Salas, Sánchez-Ferro, y Ángel, 2016).

La etiología multifactorial de la EP implica una pérdida progresiva de neuronas dopaminérgicas ubicadas en la sustancia negra pars compacta del mesencéfalo en el sistema nervioso central. Además, existe presencia de inclusiones intracelulares denominados cuerpos de Lewy, los cuales están formados por agregados insolubles de proteína alfa-sinucleina, que anormalmente se pliega y originan la enfermedad (Jankovic, 2008; Martínez-Fernández et al., 2016).

La marcha en la EP se diferencia por la presencia de un patrón rígido y acinético de movimiento, que se acentúa con el progreso de la enfermedad. Inicialmente la sintomatología motora comienza a un lado del cuerpo (hemicuerpo), luego puede extenderse del otro lado, incluso en etapas tempranas de la enfermedad (Schoneburg, Mancini, Horak, y Nutt, 2013). El caminar se vuelve poco a poco más lento, disminuye la oscilación fisiológica de la extremidad superior (balanceo) y la extremidad inferior puede arrastrarse ligeramente en el hemicuerpo afectado (Cheng, Ulane, y Burke, 2010).

En la medida que progresa la enfermedad, la marcha es notoriamente más lenta con una marcada disminución de la longitud de los pasos. La base de apoyo y de sustentación se torna más estrecha y la postura adquiere un patrón de flexión (postura encorvada), que afecta el cuello, los hombros y el tronco. Las oscilaciones de las extremidades superiores en esta etapa adquieren una posición aducida y flexionada. Así también afecta la flexión de extremidad inferior, necesaria para lograr una altura que permita dar los pasos, ocasionando que la marcha se vuelva intermitente con aumentada variabilidad de los pasos. Realizar otras tareas simultáneamente a la acción de caminar, por ejemplo, hablar, empeora las alteraciones todas estas alteraciones (Hass, Waddell, Wolf, Juncos, y Gregor, 2008; Schoneburg et al., 2013; Sveinbjornsdottir, 2016). Comúnmente también, los pacientes con EP perciben que subir escaleras en comparación con caminar es una tarea más sencilla, no así ponerse de pie desde una posición sentada, en la que se hace evidente el uso de extremidades superiores para lograrlo. Algunos 
pacientes desarrollan una propensión a inclinarse hacia adelante, aumentado la frecuencia de pasos mientras caminan, este patrón de marcha particular en EP se conoce como festinación, el cual causa riesgo de caídas, al igual que la pérdida de reflejos posturales (Schoneburg et al., 2013).

A partir de las alteraciones de la marcha que se manifiestan en los pacientes con EP, se ha desarrollado una 'cápsula formativa' que permite abordar de manera flexible estos contenidos específicos de la marcha, adaptándolos a un contexto de formación de estudiantes de medicina, que en su desempeño disciplinar se verán enfrentados a este tipo de pacientes. Las 'cápsulas formativas' constituyen actividades formativas breves, muy enfocadas y a su vez prácticas que tienen por objetivo poder ser inmediatamente aplicables de forma clara y directa (Hug, 2005). La 'capsula formativa' se ha planificado en base a un tema puntual, en este caso, reconocimiento de alteraciones y patrón de marcha en un paciente con EP, para afianzar el conocimiento y práctica en esta área.

\section{Objetivo}

Desarrollar y evaluar una Cápsula formativa sobre patrones de marcha alterados en la enfermedad de Parkinson a través de un escenario clínico.

\section{MÉTODO}

Partiendo de la metodología tradicional de resolución de casos, se generó una Cápsula formativa de 2 horas de duración, con la finalidad de reconocer las alteraciones motoras y el patrón de marcha en un escenario clínico con un paciente con enfermedad de Parkinson. La cápsula formativa fue impartida en la asignatura optativa de gerontología Envellir bé de la Universidad Autónoma de Barcelona. Contó con una participación de 11 estudiantes de segundo grado de medicina. Para evaluar su eficacia, se aplicó una prueba pre-post sobre la capacidad para identificar signos motores de la enfermedad, alteraciones y patrón general de la marcha, mediante el reconocimiento visual con apoyo de videos de una secuencia de marcha en plano frontal y sagital.

La cápsula comprendió los siguientes apartados según los lineamientos del micro aprendizaje propuesto por Hug (2005):

Se llevó a cabo en una primera instancia una evaluación inicial de conocimientos previos de la enfermedad, el cual fue medido a través de un test de preguntas abiertas y selección de sintomatología de la enfermedad.

Se realizó una exposición oral con apoyo audiovisual, donde se dieron a conocer los antecedentes de la EP según su cuadro clínico enfatizando los signos/síntomas motores. 
A continuación, se identificación de parámetros de estudio: marcha, postura y equilibrio en un sujeto adulto normal.

Posteriormente, los componentes de normalidad fueron comparados con los elementos que estaban alterados en el sujeto de estudio (caso clínico) enfatizando su marcha, postura y equilibrio.

Se registraron las observaciones clínicas de signos motores y estadio de la enfermedad en ficha de evaluación entregada a cada integrante de la audiencia.

Consecutivamente se dedicó tiempo para desarrollar un diálogo que permitiera compartir opiniones del caso estudiado. Además, se otorgó tiempo a consultas.

La identificación y cumplimentación de pautas de observación de las alteraciones de la marcha, la postura y el equilibrio fue completada de manera individual, al finalizar la revisión de los registros de videos y discusión de los hallazgos.

Con la cumplimentación de la ficha de observación se realizó la unificación de criterios y se presentaron las respuestas mediante un resumen final.

La cápsula formativa finalizó con la aplicación del instrumento de evaluación utilizado al inicio de la formación con la finalidad de medir el grado de conocimientos nuevos adquiridos posterior a la 'capsula formativa'.

La cápsula incluyó los siguientes apartados, los cuales serán expuestos en el ítem de resultados:

Búsqueda bibliográfica: se realizó una exploración de artículos, libros y revisiones científicas relevantes para el tema a tratar basadas específicamente en las alteraciones de la marcha de un paciente con EP.

Elaboración del material didáctico: en base a la bibliografía consultada, se confeccionó un contenido teórico y práctico para impartir en la cápsula formativa. Dentro del material práctico se seleccionó un escenario clave que diera a conocer la problemática a tratar.

Puntos claves a evaluar: se generaron 8 puntos clave de aprendizaje, usando como base el objetivo principal de la cápsula formativa. Así mismo, se incluyó una evaluación previa a la impartición de la cápsula formativa, y una posterior que midiera el grado de aprendizaje alcanzado sobre dichos puntos.

Resultados de aprendizaje de la cápsula formativa: en este punto se darán a conocer los principales indicadores y cambios, previa capsula formativa, aplicada a los participantes a quienes estuvo dirigido en esta ocasión. 


\section{CASTILLO-MARIQUEO y GIMÉNEZ-LLORT. Reconocimiento de alteraciones y patrón de marcha en la enfermedad de Parkinson}

\section{RESULTADOS}

Búsqueda bibliográfica. Se seleccionaron y priorizaron los contenidos a través de una búsqueda bibliográfica en las bases de datos: PubMed, Web of Science, Lilacs y Scielo. Se utilizaron los términos de búsqueda: Enfermedad de Parkinson/Parkinsons' disease; marcha/gait; equilibrio/balance; postura/posture. Los artículos relevantes fueron analizados mediante lectura crítica para su selección, para que respondiera a las necesidades teóricas y prácticas de este tema.

Material didáctico y puntos clave a evaluar. A partir de las evidencias y recomendaciones de la literatura, en esta 'cápsula formativa' se consideraron 8 puntos clave para el reconocimiento de las alteraciones y patrón de marcha en EP, siendo estos:

Inicio dubitativo: dificultad para levantar el pie y empezar a andar.

Disminución o pérdida del balanceo de los brazos. Marcha lenta arrastrando los pies.

Disminución de la zancada y longitud del paso.

Festinación: el paciente empieza a caminar cada vez más rápido, con los pasos cada vez más cortos, inclinándose hacia adelante.

Dificultad para girar mientras se camina. Episodios de bloqueo (freezing).

Disminución de la base de sustentación y apoyo.

La capsula formativa fue impartida de manera oral, apoyándose de material audiovisual, tales como videos de secuencias de marcha de un paciente filmado en plano sagital y frontal (vista anterior y posterior).

Resultados de aprendizaje de la cápsula formativa. Los participantes de la 'cápsula formativa' fueron 11 estudiantes de medicina de segundo año de carrera, con una edad promedio de 20 años. Sólo 3 de los participantes mencionaron en la evaluación conocer a una persona con EP de manera cercana.

En la evaluación previa realizada antes del desarrollo de la cápsula formativa se midieron el grado de conocimiento en la conceptualización o definición de la enfermedad, antecedentes epidemiológicos, alteraciones neurobiológicas, sintomatología motora/no motora, y alteraciones de mayor frecuencia que se presentan en la marcha de las personas con diagnóstico de EP. Respecto a la conceptualización y características epidemiológicas de la enfermedad, destacaron los conceptos: "dolencia", "enfermedad neurodegenerativa" y "enfermedad de mayor frecuencia en personas mayores". Considerando las alteraciones neurobiológicas de la enfermedad, los participantes reconocen la alteración del movimiento, como la más importante. Dentro de la identificación de sintomatología motora de la enfermedad, destaca el temblor e inestabilidad, y en la no motora la ansiedad, demencia y depresión. Cuantitativamente el $54 \%$ de los sujetos reconocían por lo menos 2 signos de la enfermedad, pero sólo un $8 \%$ de las alteraciones específicas en la marcha. En este ámbito específico de la marcha, 
objetivo de este módulo formativo es dónde efectivamente existía menor grado de conocimiento previo, inclusive presencia de errores en la utilización de conceptos y signos de las manifestaciones motoras de la enfermedad. Específicamente en el ámbito motor, eran desconocidos los estadios de progresión de esta sintomatología en la enfermedad, este ítem no fue respondido en la evaluación inicial, al igual que los hallazgos fuera del temblor y la inestabilidad postural.

Al finalizar la cápsula formativa, los participantes en su totalidad fueron capaces de reconocer los 4 signos cardinales de la EP, diferenciando el momento en el que aparecen y su progresión según la escala de severidad de Hoehn y Yahr.

La identificación de alteraciones motoras presentes en la marcha aumentó al $60 \%$, siendo los signos: rigidez, bradicinesia, dicinesia y festinación, los conceptos nuevos que incorporaron en sus respuestas. El patrón de marcha general de la EP también aumento significativamente respecto a los indicadores de alteración, desde un reconocimiento inicial promedio de 1,6 (SD: 1,7) signos a un reconocimiento de 4,9 (SD: 0,3) signos motores de EP, diferenciando signos motores, postura, características en las fases de la marcha y variabilidad espacio temporales de la marcha $\left(X^{2}=0,046\right)$.

\section{CONCLUSIONES/DISCUSIÓN}

Considerando las limitaciones del tamaño de la muestra, la capsula formativa evidenció su eficacia al mejorar significativamente la capacidad de reconocimiento e identificación de alteraciones y patrón de marcha en la enfermedad de Parkinson. Además, estimuló la participación de los estudiantes, incentivando su aportación activa en torno a un escenario real. Este método formativo breve puede ser trasladable también a la formación en enfermería, fisioterapia y terapeutas ocupacionales del ámbito de la gerontología y geriatría. Ya que, tradicionalmente las investigaciones se han enfocado en el análisis del trastorno del movimiento, principalmente en la disfunción postural que presentan los pacientes, buscando así alternativas de tratamiento para disminuir los síntomas de la enfermedad, por lo que este tipo de estrategia formativa puede constituir el nexo entre los hallazgos científicos y el desempeño clínico inmediato y real.

Por otra parte, el control de la enfermedad depende en gran medida de la terapia farmacológica, la terapia de rehabilitación física y psicológica, ya que no posee cura o tratamiento definitivo, por lo que enfatizar aspectos concretos y específicos, que refuercen mediante la capacitación y formación continua, los objetivos de rehabilitación apuntan directamente a mejorar la calidad de vida y el bienestar, así como aumentar la funcionalidad y mantener la independencia de quien padece esta enfermedad.

Los trastornos motores son la principal afección en la EP, caracterizados por una discapacidad progresiva en la realización de actividades de la vida diaria, y una alteración del estado funcional (Orcioli-Silva et al., 2017). La postura corporal dentro de 
los componentes motores relevantes de la EP, es difícil de reconocer de forma integrada en la ejecución de la marcha, ya que el trastorno más evidente es el temblor (Schoneburg et al., 2013). Junto al análisis postural en la marcha es necesario identificar las fases de ésta y el movimiento considerado normal para cada segmento corporal, ya que permite apreciar las alteraciones focalizando elementos clave como la alteración en los apoyos, altura y longitud de los pasos al caminar (Cerda, 2010).

La identificación de las alteraciones en el control del equilibrio tanto en posición estática como dinámica, acompañado de un adecuado control postural frente al cambio de posiciones y como éstos son necesarios en la locomoción para detectar caídas y alteraciones en la variabilidad de la marcha, hacen necesaria una evaluación exhaustiva de todos los componentes antes mencionados, y propone la creación de nuevas estrategias terapéuticas para su rehabilitación que pueden nacer desde capacitaciones específicas con énfasis práctico en todos los ámbitos de acción que posee el proceso de rehabilitación de esta enfermedad.

\section{Agradecimientos \\ UAB2019/GE260408, EB (Associació Envellir bé - Saber envejecer) 2018/1601 - CONICYT/BECAS CHILE/72180026}

\section{REFERENCIAS}

Cerda, A.L. (2010). Evaluación del paciente con trastorno de la marcha. Revista del Hospital Clínico de la Universidad de Chile, (21), 326-336.

Cheng, H.C., Ulane, C., y Burke, R. (2010). Clinical progression in Parkinson's disease and the neurobiology of Axons. Annals of Neurology, 67(6), 715-725. doi: 10.1002/ana.21995.Clinical

Hass, C.J., Waddell, D.E., Wolf, S.L., Juncos, J.L., y Gregor, R.J. (2008). Gait initiation in older adults with postural instability. Clinical Biomechanics, 23(6), 743-753. doi: 10.1016/j.clinbiomech.2008.02.012.GAIT

Hug, T. (2005). Micro Learning and Narration: Exploring possibilities of utilization of narrations and storytelling for the designing of "micro units" and didactical micro-learning arrangements. Proceedings of the Fourth Media in Transition Conference.

Jankovic, J. (2008). Parkinson's disease: Clinical features and diagnosis. Journal of Neurology, Neurosurgery and Psychiatry, 79(4), 368-376. doi: 10.1136/jnnp.2007.131045

Martínez-Fernández., R., Gasca-Salas C., C., Sánchez-Ferro, Á., y Ángel Obeso, J. (2016). Actualización en la enfermedad de parkinson. Revista Médica Clínica las Condes, 27(3), 363-379. doi: 10.1016/j.rmclc.2016.06.010

Orcioli-Silva, D., Barbieri, F.A., Simieli, L., Vitorio, R., Santos, P.C.R., Beretta, V.S., y Gobbi, L.T.B. (2017). Walking behavior over multiple obstacles in people with Parkinson's disease. Gait and Posture, 58, 510-515. doi: 10.1016/j.gaitpost.2017.09.021

Schoneburg, B., Mancini, M., Horak, F., y Nutt, J.G. (2013). Framework for understanding balance dysfunction in Parkinson's disease. Movement Disorders, 28(11), 1474-1482. doi: $10.1002 / \mathrm{mds} .25613$ 
CASTILLO-MARIQUEO y GIMÉNEZ-LLORT. Reconocimiento de alteraciones y patrón de marcha en la enfermedad de Parkinson

Suzuki, K., Okuma, Y., Uchiyama, T., Miyamoto, M., Sakakibara, R., Shimo, Y., ... Hirata, K. (2017). Impact of sleep-related symptoms on clinical motor subtypes and disability in Parkinson's disease: A multicentre cross-sectional study. Journal of Neurology, Neurosurgery and Psychiatry, 88(11), 953-959. doi: 10.1136/jnnp-2017-316136

Sveinbjornsdottir, S. (2016). The clinical symptoms of Parkinson's disease. Journal of Neurochemistry, 139, 318-324. doi: 10.1111/jnc.13691

Recibido: 23 de septiembre de 2019 Recepción Modificaciones: 07 de octubre de 2019

Aceptado: 09 de octubre de 2019 